\title{
Crianças prematuras: caracterização e intervenção fisioterapêutica
}

\author{
Premature children: characterization and \\ physical therapy intervention
}

Gisélia Gonçalves de Castro,' Glória Lúcia Alves Figueiredo, ${ }^{1}$ Maria Aparecida Tedeschi Cano Universidade de Franca (Unifran), Franca, SP, Brasil.

Recebido em: 26/07/2016 / Aceito em: 30/08/2016 / Publicado em: 30/09/2016

giseliagcastro@gmail.com

\section{RESUMO}

Objetivo: determinar as características clínicas dos recém-nascidos prematuros de um município do interior de Minas Gerais e a intervenção fisioterapêutica destes recém-nascidos que foram encaminhados para esse serviço, após a alta hospitalar. Método: foi realizado um estudo descritivo exploratório, a partir de dados secundários do SINASC (Sistema de Informações sobre Nascidos Vivos), Declaração de Óbitos da Vigilância Epidemiológica e prontuários das Unidades Básicas de Saúde. A análise estatística dos fatores associados ao óbito foi realizada, utilizando-se o teste do $\chi 2$ para comparação das variáveis qualitativas e o teste exato de Fisher. A medida de associação entre o óbito e as demais variáveis foi determinada, através da estimação do risco relativo. Resultados: os nascidos vivos corresponderam a um total de 1.203, sendo $1.144(95,0 \%)$ a termo e 59 $(5,0 \%)$ pré-termo, com o peso $\leq$ que $2500 \mathrm{~g}$ e idade gestacional < que 37 semanas. Destes últimos, 51 nasceram em hospital público e oito em instituições privadas. A maior frequência de RNPT foi entre 32 a 36 semanas (60,8\%). Ocorreram doze óbitos entre os prematuros, sendo que a classe de 28 a 31 semanas, que corresponde à prematuridade moderada, foi a que apresentou maior número de óbitos $(47,4 \%)$. Com relação à mortalidade, houve diferença significante $(p<0,01)$ entre os recém-nascidos que apresentaram um Apgar $f 7$ no $1^{\circ}$ minuto $(R R=11,79)$ e no $5^{\circ}$ minuto $(R R=18,11)$. Considerações finais: apenas cinco recém-nascidos pré-termo foram encaminhados para intervenção fisioterapêutica e apresentavam tempo de gestação $\geq 28$ semanas de desenvolvimento, representando $12,8 \%$ da amostra. Um atendimento sistematizado poderia proporcionar a detecção precoce e intervenções eficazes, a partir de estímulos adequados e preservação do desenvolvimento neuromotor, especialmente para os recém-nascidos prematuros de prematuridade extrema, que não foram encaminhados para estimulação do desenvolvimento, dado que perpassa como intervenção promissora para melhoria da qualidade de vida desta população. Palavras-chave: Recém-nascido; Prematuridade; Fisioterapia; Promoção de Saúde.

\section{ABSTRACT}

Objective: determine the clinical characteristics of premature newborns from a city of Minas Gerais state, and physiotherapy intervention of these premature newborns that were referred to this service, after hospital discharge. Method: this is a descriptive exploratory study from secondary data of SLBI (System on Live Births Information) Statement of deaths of Epidemiological Surveillance and records of the Basic Health Units. The statistical analysis of factors associated with death was performed using the qui-square test to compare qualitative variables and Fisher's exact test. The measure of association between death and other variables was determined using the relative risk estimation. Results: live births correspond to a total of 1,203 being 1,144 (95.0\%) term and 59 (5.0\%) preterm, with weight $\leq$ than 2,500 $\mathrm{g}$ and gestational age $<37$ weeks. Of these latest, 51 were born in a public hospital and eight in private institutions. The highest incidence of preterm was between 32 to 36 weeks (60.8\%). There were twelve deaths among premature infants, considering the class from 28 to 31 weeks which corresponds to moderate prematurity showing the highest number of deaths $(47.4 \%)$. Regarding mortality there is a significant difference $(p<0.01)$ between the newborns who had an Apgar $£ 7$ in $1^{\circ}$ minute $(R R=11.79)$ and in $5^{\circ}$ minute $(R R=18.11)$. Closing remarks: only five preterm infants were referred to the physical therapy 
intervention and had gestational time $\geq 28$ weeks of development, representing $8.5 \%$ of the sample. A systemized care could provide early detection and effective interventions, from appropriate stimuli and preservation of neuromotor development, especially for preterm of extreme prematurity that were not referred for stimulation of development, data that represents a promising intervention for improving the quality of life of this population.

Keywords: Newborn; Prematurity; Physiotherapy; Health Promotion.

\section{INTRODUÇ̃̃O}

A criança que nasce prematura, seja em decorrência do menor tempo de gestação e/ou com baixo peso poderá ter anormalidades neurológicas transitórias, envolvendo postura, habilidades motoras finas e grosseiras, coordenação do equilíbrio, reflexos e principalmente distonias. Estas intercorrências clínicas surgem em virtude da imaturidade do recém-nascido.

Segundo a Organização Mundial de Saúde (OMS), um bebê imaturo ou chamado pré-termo é aquele que nasce antes de completar 37 semanas de idade gestacional. De acordo com Formiga, et al., ${ }^{2}$ a criança antes do termo, tanto pode ser denominada de "pré-termo", quanto de "prematuro". Os recém-nascidos pré-termo (RNPT) podem ser classificados de acordo com a idade gestacional, quanto ao peso de nascimento e ainda quanto à adequação do peso de nascimento à idade gestacional. ${ }^{3-5}$ A Idade gestacional é classificada de 32 semanas a 36 semanas, prematuridade acentuada, com 28 semanas a 31 semanas de idade gestacional e prematuridade extrema, quando a idade gestacional é inferior a 28 semanas. ${ }^{6}$

No entanto, no primeiro ano de vida, especial atenção deve ser dada à evolução motora do prematuro, com avaliação do tônus, postura, mobilidade ativa e força muscular. Diferente da criança que nasceu a termo, ou seja, com nove meses de gestação, a criança prematura tem peculiaridades que a torna passível de observação mais cuidadosa, especialmente com relação a seu desenvolvimento motor. Tal fato deve ser observado não apenas no hospital, na unidade intensiva, mas, principalmente após a alta, no acompanhamento nas Unidades Básica de Saúde. ${ }^{1,7}$

É de extrema importância que se faça a avaliação, o acompanhamento e a intervenção o mais precoce possível no desenvolvimento neuromotor dos bebês prematuros, sem que se espere chegar a uma determinada idade para o início desta intervenção, ou ao se constatar que a criança não desempenha determinada função motora para a idade. A intervenção deve ser realizada logo que se detectem as anormalidades no desenvolvimento do bebê prematuro. ${ }^{8,9} \mathrm{O}$ fisioterapeuta atua com crianças com diversas alterações neuropsicomotoras, sendo um conhecedor do desenvolvimento normal e patológico o seu desafio é avaliar e compreender precisamente qualquer atraso que saia dos limites normais do desenvolvimento. ${ }^{7,9}$

Esta pesquisa buscou determinar as características clínicas e demográficas dos recém-nascidos prematuros de um município mineiro e a intervenção fisioterapêutica nos RNPT que foram encaminhados para esse serviço, após a alta hospitalar.

\section{MÉTODO}

Para atingir o objetivo proposto foi realizado um estudo descritivo exploratório a partir de dados secundários do SINASC (Sistema de Informações sobre Nascidos Vivos), Declaração de Óbitos (DO) da Vigilância Epidemiológica e dados das Unidades Básicas de Saúde sobre o encaminhamento e intervenção fisioterapêutica do recém-nascido prematuro. No ano de 2004, no município de Patrocínio, interior de Minas Gerais, para avaliação do desenvolvimento neuro motor.

O SINASC é um sistema de informações de âmbito nacional, sob responsabilidade das Secretarias Estaduais e Municipais de Saúde, concebido para diminuir a subnotificação e melhorar a qualidade de informação sobre nascidos vivos. Este sistema é alimentado pelas declarações de nascido vivo (DNV), documento oficial emitido pelo hospital, onde ocorre o nascimento. ${ }^{10}$

A população em estudo, pesquisado no referido sistema, constituiu-se de nascidos vivos prematuros com idade gestacional menor que 37 semanas e peso ao nascimento igual ou inferior a $2.500 \mathrm{~g}$, nascidos no Hospital Santa Casa de Misericórdia, em Patrocínio, município do interior de Minas Gerais. A escolha de analisar dados da DNV emitidos por este hospital foi pelo fato de ser um hospital que atende pelo Sistema Único de Saúde (SUS) e o acompanhamento clínico destas crianças é realizado nas Unidades Básicas de Saúde (UBS), pelo Programa Saúde da Criança.

A análise estatística dos fatores associados ao óbito foi realizada utilizando-se o teste do $\mathrm{c}^{2}$ para comparação entre os valores percentuais (variáveis qualitativas) quando o $\mathrm{n}$, na tabela $2 \times 2$, for maior que 5 e o teste exato de Fisher, quando o $n$ for igual ou menor que cinco. A medida de associação entre o óbito e as demais variáveis foi determinada, através da estimação do risco relativo. A significância estatística foi definida por um valor de $p$ menor que 0,05. A análise das variáveis foi realizada, utilizando-se os programas estatísticos SPSS PC versão 11.0 (SPSS, Chicago) e o Epi Info Software versão 2000 (CDC, Atlanta).

Após a análise dos dados da população em estudo, com base no endereço da mãe informado pela declaração de nascidos vivos, foram feitas visitas às dez UBS do município, com o objetivo de verificar o encaminhamento e intervenção fisioterapêutica das crianças nascidas prematuras para tratamento neuromotor.

\section{RESULTADOS}

Os nascidos vivos em Patrocínio/MG, no ano de 2004, corresponderam a um total de 1.203, sendo $1.144(95,0 \%)$ a termo e $59(5,0 \%)$ pré-termo, com o peso $\leq$ que $2500 \mathrm{~g}$ e idade gestacional < que 37 semanas. Deste total de nascidos vivos pré-termo, 51 nasceram no Hospital Santa Casa de Misericórdia N.S. do Patrocínio, atendidos pelo SUS. Destes 51 nascidos vivos pré-termos, 12 foram ao óbito, totalizando uma amostra de 39 recém-nascidos prematuros. 
Com relação ao tempo de gestação, a maior frequência de bebês prematuros foi entre 32 a 36 semanas $(60,8 \%)$. No que se refere ao número de óbitos, em todas as classes de tempos de gestação, foi de quatro óbitos, totalizando 12 óbitos. Relacionando o número de óbitos com os prematuros nas três classes de tempo de gestação, observou-se que a classe de 28 a 31 semanas, que corresponde à prematuridade moderada, foi a que apresentou maior número de óbitos, correspondendo a $47,4 \%$ (Tabela 1 ).

Tabela 1 - Distribuição de Óbitos de RNPT em cada idade gestacional nascidas em Patrocínio/MG.

\begin{tabular}{lcccc}
\hline \multirow{2}{*}{ Tempo de gestação } & \multicolumn{2}{c}{ Prematuros } & \multicolumn{2}{c}{ Óbitos } \\
& Fa & Fr & Fa & Fr \\
\hline 22 a 27 semanas & 11 & 21,6 & 4 & 38,8 \\
28 a 31 semanas & 9 & 17,6 & 4 & 47,4 \\
32 a 36 semanas & 31 & 60,8 & 4 & 13,7 \\
Total & 51 & 100 & 12 & 100 \\
\hline N=Fa (Frequência absoluta): \% $=$ Fr (Frequência relativa) & \multicolumn{3}{l}{}
\end{tabular}

No que diz respeito à mortalidade, houve diferença significativa $(p<0,01)$ entre os recém-nascidos que apresentaram um Apgar $£ 7$ no $1^{\circ}$ minuto $(R R=11,79)$ e no $5^{\circ}$ minuto $(R R=18,11$ ) (Tabela 2$)$.

Dos 39 RNPT sobreviventes, cinco crianças foram encaminhadas para a intervenção fisioterapêutica, representando $12,8 \%$ da amostra. Destas cinco crianças, duas residiam em bairro de periferia do município e foram encaminhadas para o setor de fisioterapia implantado na UBS do próprio bairro. Três RNPT foram encaminhadas Associação de Pais e Amigos dos Excepcionais de Patrocínio, uma instituição filantrópica do município, uma vez que as UBS próximas as suas residências não possuíam atendimento fisioterapêutico.

Esses RNPT que foram encaminhados para inter- venção fisioterapêutica apresentavam tempo de gestação $\geq 28$ semanas. Ressalta-se, que no grupo dos RNPT com $\leq 27$ semanas, que corresponde à prematuridade extrema, nenhuma das crianças foi encaminhada para tratamento fisioterapêutico.

Com relação a idade materna, não se detectou diferença significativa entre óbitos de RNPT para mães com idade $\leq 19$ anos. No entanto, houve uma associação entre o óbito e idade materna $\geq 35$ anos $(p=0,023 ;$ R.R. $=3,14)$ (Tabela 3).

\section{DISCUSSÃO}

Como já referido no presente estudo, os nascimentos de prematuros no município de Patrocínio/MG, da região sul do Brasil, correspondem a $5 \%$ dos nascidos, confirmando os maiores índices de prematuridade na região Sudeste e Sul, seguidas pelo Centro Oeste e por último o Norte..$^{10,11}$ Estudos realizados por França e Lask ${ }^{12}$ mostraram a proporção de nascimentos pré-termos mais elevada na cidade de Pelotas (Rio Grande do Sul),16\%, seguida de 10,8\% no Rio de Janeiro (Rio de Janeiro).

Já, em países desenvolvidos como os Estados Unidos e a Dinamarca há uma taxa elevada de prematuridade, o que predispõe os neonatos a maiores riscos de óbito por fatores diversos. ${ }^{12,13}$

Segundo Kilsztajn ${ }^{14}$ e Ribeiro, ${ }^{15}$ o parto prematuro está associado a fatores sócio-econômicos. No presente estudo verificou-se que dos 59 RNPT, 51 nasceram no hospital conveniado pelo SUS, correspondendo a $86,4 \%$ dos partos, sendo que a porcentagem dos nascidos em hospitais particulares da cidade foi de 0,7\%. A análise dos grupos populacionais compostos pelos prematuros residentes em bairros pobres da cidade mostrou o nível sócio-econômico da população em estudo como fator de risco para prematuridade. Indica-se que as diferenças regionais nas taxas de baixo peso ao nascimento no país parecem estar mais relacionadas à disponibilidade

Tabela 2 - Distribuição da nota do Apgar do RNPT com o óbito e não óbito.

\begin{tabular}{|c|c|c|c|c|c|}
\hline $\begin{array}{l}\text { Apgar } \\
\text { (nota) }\end{array}$ & & $\begin{array}{c}\text { Óbito } \\
\mathrm{N}=12(\%)\end{array}$ & $\begin{array}{l}\text { Não Óbito } \\
\text { N=39 (\%) }\end{array}$ & $\begin{array}{c}\text { Valor } \\
\text { (p) }\end{array}$ & RR (IC) \\
\hline \multirow{2}{*}{$1^{\circ}$ minuto } & $>8$ & $0(0 \%)$ & $24(61,53 \%)$ & 0,0005 & $11,79(1,62-82,67)$ \\
\hline & f7 & $12(100 \%)$ & $15(38,46 \%)$ & & \\
\hline \multirow[t]{2}{*}{$5^{\circ}$ minuto } & $>8$ & $1(8,33 \%)$ & $30(76,92 \%)$ & 0,0003 & $18,11(2,38-122,07)$ \\
\hline & f 7 & $11(91,66 \%)$ & $9(23,07 \%)$ & & \\
\hline
\end{tabular}

RR= Risco Relativo; IC = Intervalo de Confiança

Tabela 3 - Associação da idade materna do RNPT com o óbito do RNPT.

\begin{tabular}{lccc}
\hline $\begin{array}{l}\text { Idade materna } \\
\text { anos }\end{array}$ & $\begin{array}{c}\text { Óbito } \\
\mathbf{N}=12(\%)\end{array}$ & $\begin{array}{c}\text { Não Óbito } \\
\mathbf{N}=39(\%)\end{array}$ & $\begin{array}{c}\text { Valor } \\
(\mathbf{p})\end{array}$ \\
\hline$<19$ & $3(16,67)$ & $24(83,33)$ & 0,39 \\
$\geq 19$ & $9(27,27)$ & & $3(42,86)$ \\
$\geq 35$ & & $36(81,82)$ & 0,023 \\
$<35$ & $4(57,14)$ & $3(18,18)$ & \\
\hline
\end{tabular}

$R \cdot R=0,61$ (I.C. $=0,19-1,98) ; \quad R \cdot R=3,14(I . C .=1,28-7,71)$. 
de assistência perinatal do que às condições sociais ${ }^{16}$. Estudos realizados por Chalem et al. (2007), ${ }^{17}$ apontaram que renda familiar menor ou igual a um salário mínimo e o nascimento em hospital público foram os fatores de risco para prematuridade, corroborando com alguns estudos de Scochi ${ }^{18}$ e Araujo ${ }^{19}$ que consideraram a baixa renda um fator predisponente a partos prematuros e alterações no desenvolvimento motor.

Quanto aos óbitos entre os prematuros da pesquisa, observou-se uma elevada porcentagem, 47,7\%, no grupo nascidos com idade gestacional de 28 a 31 semanas. Este resultado está de acordo com estudos de autores que relatam neste grupo a taxa de mortalidade é elevada, em decorrência da doença da membrana hialina grave, citada como a responsável pelos óbitos. ${ }^{3,13,15}$

Outro aspecto que é importante ressaltar refere-se à idade gestacional, relacionada a problemas de ordem neurológica. Autores como $\mathrm{Hack}^{20}$ e Giachetta et al. ${ }^{1}$ afirmam que, quanto menor a idade gestacional, menor o peso ao nascimento, maior a probabilidade de problemas neurológicos ou desvios no desenvolvimento subsequente. A maior prevalência de BPN no Brasil foi de $10,4 \%$ no ano $1996 .{ }^{21}$ A prevenção do baixo peso ao nascimento, avaliando-se ao nascimento pré-termo, assim como a identificação dos fatores modificáveis por intervenções sociais de saúde e nutrição, deve constituir o alvo das intervenções destinadas a um melhor impacto na saúde materno-infantil. Nesse sentido, observa-se um aumento crescente na proporção de partos prematuros no Brasil, associado à maior ocorrência de baixo peso ao nascer. ${ }^{22}$ Há necessidade de se detectar precocemente alterações neuro-motoras das crianças prematuras, principalmente os de baixo peso e intervir imediatamente.

Nos neonatos pré-termos encaminhados à intervenção precoce, a avaliação fisioterapêutica, detecta se há necessidade de tratamento terapêutico visando maior qualidade de vida dos recém-nascidos pré- termos. ${ }^{23}$ Assim, o presente estudo mostrou que dos 39 RNPT, apenas cinco (12,8\%) foram encaminhados para acompanhamento fisioterapêutico, já nos recém-nascidos prematuros extremos nenhum foi encaminhado, portanto, seria importante considerar a assistência pós-natal destas crianças.

Outra variável que apresentou forte associação à morte foi o índice de Apgar, tanto no primeiro minuto, quanto no quinto minuto com nota inferior a sete. De acordo com estudos de Oliveira et al. ${ }^{24}$ RNPT tem grande probabilidade a apresentar Apgar de 0 a 3, agravando o quadro, podendo estar associado ao óbito. Em conformidade com o estudo, Geib et al. ${ }^{25}$ evidenciou que os nascidos vivos com pontuação de Apgar inferior a sete, no quinto minuto de vida demonstraram um risco de óbito 8,7 vezes maior do que os nascidos com pontuação superior a sete.

Segundo pesquisas de Silveira e Enumo ${ }^{26}$ e de Araújo ${ }^{18}$ e Almeida ${ }^{27}$ a idade materna foi um fator de risco importante para o baixo peso e mortalidade infantil, particularmente entre as adolescentes com menos de 20 anos e entre as mães com 35 anos ou mais. Estudos realizados em Fortaleza (Ceará), por Nascimento et al. ${ }^{28}$ evidenciou a idade materna como fator predisponente à prematuridade, assinalando como responsável por
10,6\% dos óbitos de recém-nascido advinham de mães adolescentes e que $15 \%$ de mães com idade superior a 35 anos ou mais. Neste estudo, o risco significativo para óbito de recém-nascido foi o grupo de mães com $\geq 35$ anos, com probabilidade de óbito de 3,14. Estes dados estão em discordância com estudo realizado em São Luís (Maranhão), em que as maiores taxas de mortalidade infantil foram observadas no grupo de adolescentes com menos de 18 anos. ${ }^{29}$

A preocupação com a qualidade de vida e com os aspectos que envolvem a sobrevida dos RNPT deve ser uma preocupação dos profissionais de saúde. Diversos autores, entre eles, Lopes ${ }^{5}$ e Reis ${ }^{30}$ relatam que este grupo tem maior chance de paralisia cerebral, retardo mental, dificuldades motoras, visuais e auditivas. Além disso, autores relatam que a intervenção fisioterapêutica deve ser aplicada ao bebê de alto risco, o mais precoce possível para que ele possa desenvolver habilidades normais. ${ }^{31-35}$

\section{CONSIDERAÇÕES FINAIS}

Os resultados indicam que os RNPT nascidos no hospital público do município avaliado apresentaram peso inferior a $2500 \mathrm{~g}$, podendo estar associados a provenientes de famílias residentes em bairros periféricos da cidade e, predominando a idade gestacional menor que 37 semanas, apgar menor que sete e idade materna maior que 35 anos.

Embora a prematuridade seja um risco para o desenvolvimento neuro motor infantil, esta pesquisa revelou baixo número de encaminhamentos para intervenção fisioterapêutica precoce, logo após a alta hospitalar. Seriam necessários estudos para identificar as razões pelas quais os RNPT, especialmente os de prematuridade extrema não foram encaminhados para estimulação precoce, uma vez que a mesma poderia melhorar a qualidade de vida desta população.

No entanto, destaca-se ser imprescindível a adoção de medidas mais efetivas nos serviços públicos de saúde, que garantam um atendimento mãe-filho desde o período pré-natal, neonatal e pós-natal principalmente, no que diz respeito à promoção da saúde dos recém-nascidos. Um atendimento sistematizado poderia proporcionar a detecção precoce e intervenções eficazes, a partir de estímulos adequados e preservação do desenvolvimento neuromotor, especialmente para os recém-nascidos prematuros.

\section{REFERÊNCIAS}

1. Giachetta L, Nicolau CM, Costa APBM da, Zuana AD. Influência do tempo de hospitalização sobre o desenvolvimento neuromotor de recém-nascidos pré-termo. Fisioter Pesqui 2010;17(1):24-29. doi: 10.1590/S180929502010000100005.

2. Formiga, CKMR. Comparison of the motor development in preterm infants from two brazilian regional samples. Rev Bras de Crescimento e Desenvolv Hum. São Paulo 2013;23(3):352-356.

3. Lissauer T, Graham C. Manual ilustrado de pediatria. 2 ed. Rio de Janeiro: Guanabara koogan, 2003. 
4. Lamônica DACL, Picolini MM. Habilidades do desenvolvimento de prematuros. Rev CEFAC 2009;11(2):145-153. doi: 10.1590/S1516-18462009005000019.

5. Lopes MMCO. Avaliaçâo do desenvolvimento neuromotor da criança de risco aplicando Harris Infant Neuromotor Test (HINT), 2011; 141f. [Tese] - Universidade Federal do Ceará, Fortaleza.

6. Lorena SHT, Brito JMS. Estudo retrospectivo de crianças pré-termo no Ambulatório de Especialidades Jardim Peri-Peri. Arq Bras Oftalmol 2009;72(3):360-4. doi: 10.1590/S000427492009000300015

7. Tecklin JS. Fisioterapia Pediátrica. Porto Alegre : Artmed, 2002; 3.ed.. 479p.

8. Fernandes LV, Goulart AL, Santos AMN, Barros MC de M, Guerra CC, Kopelman BI. Avaliação do neurodesenvolvimento de prematuros de muito baixo peso ao nascer entre 18 e 24 meses de idade corrigida pelas escalas de Bayley III. J Pediatr. Rio de Janeiro 2012;88(6):471-8. doi: 10.1590/S0021-75572012000600005.

9. Maccari GM, Liberali J, In: Lanza FC (Org.). Fisioterapia em Pediatria e Neonatologia: da UTI ao Ambulatório. São Paulo: Roca 2013;6:129-157.

10. Pedraza DF, Souza MM de, Cristóvão FS, França ISX de. Baixo peso ao nascer no Brasil: revisão sistemática de estudos baseados no sistema de informações sobre nascidos vivos. Ver Atenç Saúd. São Paulo. Fev 2014;50:(2):51-64. doi: 10.13037/rbcs.vol12n41.2237.

11. Matijasevich A, Silveira MF da, Matos ACG, Neto DR, Fernandes RM, Maranhão AG, Escalante JJC, Barros FC, Victora CG. Estimativas corrigidas da prevalência de nascimentos pré-termo no Brasil, 2000 a 2011. Epidemiol Serv Saúde. Brasília 2013;22(4):557-64. doi: 10.5123/ S1679-49742013000400002.

12. França $\mathrm{E}$, Lansky S. Mortalidade infantil neonatal no Brasil: situação, tendências e perspectivas. In: Rede Interagencial de Informação para a Saúde (RIPSA). Demografia e saúde: contribuição para análise de situação e tendências. Brasília: Organização Pan-Americana da Saúde 2009;83-112.

13. Silveira MF, Matijasevich A, Horta BL, Bettiol H, Barbieri MA, Silva AA, et al.. Prevalência de nascimentos pré-termo por peso ao nascer: Revisão sistemática. Rev Saúde Pública, São Paulo 2013;47(5):992-1000. doi: 10.1590/S0034 8910.2013047004997.

14. Kilsztajn S, Rossbach AC. Assistência pré-natal, baixo peso e prematuridade no estado de São Paulo. Rev de Saúde Pública 2003;37(3):303-10. doi: 10.1590/S0034 89102003000300007.

15. Ribeiro $A M$, Guimarães MJ, Lima M de C, Sarinho SW, Coutinho SB. Fatores de risco para mortalidade neonatal em crianças com baixo peso ao nascer. Rev Saúde Pública, São Paulo 2009;43(2):246-55. doi: 10.1590/S0034 89102009005000004.

16. Silva AAM, Silva LM, Barbieri MA, Bettiol $H$, Carvalho LM, Ribeiro VS, et al. The epidemiologic paradox of low birth weight in Brazil. Rev Saúde Pública 2010;44(5):767-75. doi: 10.1590/S0034-89102010005000033.

17. Chalem E, Mitsuhiro SS, Ferri CP, Barros MCM, Guinsburg R, Laranjeira R. Gravidez na adolescência: perfil sóciodemográfico e comportamental de uma população da periferia de São Paulo, Brasil. Cad. Saúde Pública, Rio de Janeiro 2007;23(1):177-186. doi: 10.1590/S0102$311 \times 2007000100019$.

18. Scochi CGS, Ferreira FY, Góes FSN, Fujinaga Cl, Ferecini GM,
Leite AM. Alimentação láctea e prevalência do aleitamento materno em prematuros durante internação em um hospital amigo da criança de Ribeirão Preto-SP, Brasil.Ciência, Cuidado e Saúde 2008;(supl. 1)7(2):145-154. doi: 10.4025/ cienccuidsaude.v7i2.4992.

19. Araújo DMR. Fatores associados ao estado nutricional gestacional e Desfechos perinatais em usuárias do Sistema Único de Saúde (SUS), em dois municípios do estado do Rio de Janeiro. Tese (Doutorado em Ciências na área de Epidemiologia em Saúde Pública) - Escola Nacional de Saúde Pública Sérgio Arouca, Rio de Janeiro. 2012; $145 f$.

20. Hack M, Fanaroff AA. Outcomes of children extremely low birthweitgth and gestational age in the 1990s. Semin neonatal 2000;5(2):89-106. doi: 10.1053/siny.1999.0001.

21. Blanc AK, Wardlaw T. Monitoring low birth weight: an evaluation of international estimates and an updated estimation procedure. Bull World Health Organ 2005; 83(3):178-85. doi: S0042-96862005000300010.

22. Barros FC, Barros AJD, Villar J, Matijasevich A, Domingues MR, Victora CG. How many low birthweight babies in low- and middle income countries are preterm? Rev Saúde Pública 2011;45(3):607-16. doi: 10.1590/S003489102011005000019.

23. Gaetan EM, Moura-Ribeiro MVL. Developmental study of early posture control in preterm and fullterm infants. Arq. Neuro-Psiquiatr 2002;60(4). doi: 10.1590/S0004282X2002000600012.

24. Oliveira TG, Freire PV, Moreira, FT, Moraes $J$ da $S$ B de, Arrelaro RC, Rossi S, Ricardi VA, Juliano $\mathrm{Y}$, Novo NF, Bertagnon JRD. Escore de Apgar e mortalidade neonatal em um hospital localizado na zona sul do município de São Paulo. Einstein, São Paulo 2012;10(1):22-8. doi: 10.1590/S167945082012000100006.

25. Geib LTC, Fréu CM, Brandão M, Nunes ML. Determinantes sociais e biológicos da mortalidade infantil em coorte de base populacional em Passo Fundo, Rio Grande do Sul. Ciênc Saúde Coletiva 2010;15(2):363-70. doi: 10.1590/S141381232010000200011.

26. Silveira KA, Enumo SRF. Riscos biopsicossociais para o desenvolvimento de crianças prematuras e com baixo peso. Paidéia (Ribeirão Preto), São Paulo 2012;22(53):335-45. doi: 10.1590/S0103-863X2012000300005.

27. Almeida TSO, Lins RP, Camelo ADL, Mello DCCL de. Investigação sobre o $\mathrm{s}$ fatores de risco da prematuridade: uma revisão sistemática. Revista Brasileira de Ciência da Saúde 2013;17(3):301-8.

28. Nascimento R.M, Leite ÁJM, Almeida NMGS de, Almeida PC de, Silva CF da. Determinantes da mortalidade neonatal: estudo caso-controle em Fortaleza, Ceará, Brasil. Cad. Saúde Pública, Rio de Janeiro 2012;28(3):559-572. doi: 10.1590/ S0102-311X2012000300016.

29. Simões VMF, Silva AA, Bettiol H. Características da gravidez na adolescência em São Luis. Rev de Saúde Pública, São Paulo 2003;37(5):559-65. doi: 10.1590/S003489102003000500003.

30. Reis ABR, Melo RR, Morsch DS. Desempenho mental de bebes pré termo de muito baixo peso ao nascer: avaliação da estabilidade nos dois primeiros anos vida e fatores associados ao desempenho mental. Rev Bras Epidemiol. São Paulo 2012;15(1):13-24. doi: 10.1590/S1415$790 \times 2012000100002$.

31. Effgen S. Fisioterapia Pediátrica: Atendendo às necessidades das crianças. Rio de Janeiro: Guanabara Koogan, 2007. 
32. Nicolau CM, Costa APBM, Hazime HO, Krebs VeraLJ Desempenho Motor Em Recém-Nascidos Pré-Termo De Alto Risco. Rev Bras Cresc Desenvol Hum. São Paulo 2011;21(2):327.

33. Lanza FC, Gazzotti MR, Palazzi. Fisioterapia em Pediatria e Neonatologia: da UTI ao Ambulatório. São Paulo: Roca, 2013.

34. Medeiros JKB, Zanin RO, Alves KS. Perfil do desenvolvimento motor do prematuro atendido pela Fisioterapia. Rev Bras Clín Med, Londrina 2009;7:367-72.
35. Oberg GK, Campbell SK, Girolami GL, Ustad T, Jorgensen L, Kaaresen PI. Study protocol: an early intervention program to improve motor outcome in preterm infants: a randomized controlled trial and a qualitative study of physiotherapy performance and parental experiences. BMC Pediatr 2012:12:15. doi: 10.1186/1471-2431-12-15.

Como citar: CASTRO, Gisélia Gonçalves de; FIGUEIREDO, Glória Lúcia Alves; CANO, Maria Aparecida Tedeschi. Crianças prematuras: caracterização e intervenção fisioterapêutica. Cinergis, Santa Cruz do Sul, v. 17, n. 3, set. 2016. ISSN 2177-4005. Disponível em: <https://online.unisc.br/seer/index.php/cinergis/article/view/8069>. Acesso em: 11 out. 2016. doi:http:// dx.doi.org/10.17058/cinergis.v17i3.8069. 